\title{
Optimizing the property of cross-linked carbazole-based polymer for efficient hole transport in bulk-heterojunction photovoltaic device
}

\author{
Shienna Marie Pontillas ${ }^{1}$, Menandro Marquez ${ }^{1}$, Florentino C. Sumera ${ }^{1 *}$ and Rigoberto C. Advincula ${ }^{2}$ \\ ${ }^{1}$ Institute of Chemistry, University of the Philippines, Diliman, Quezon City \\ ${ }^{2}$ Case Western Reserve University, 2100, Adelbert Road, Kent Hale Smith Bldg., Cleveland, Ohio, 44106, \\ U.S.A.
}

\begin{abstract}
Thin films made of cross-linked carbazole pendants of poly(ethylmethacrylate) deposited on indium tin oxide (ITO) was assessed for potential use as hole transport layer (HTL) in a fabricated photovoltaic cell configuration (ITO/PCDTBT/PCBM/Al). Before measurement, the film was designed to have uniform molecular weight, cross-linked carbazole for high electron mobility, high transmittance at the visible wavelength range and smoothness in morphology. Results showed that the film provided 70 times more of current value at open circuit voltage than without the film under light illumination, at optimized thickness from 15 to 25 cycles of deposition at $25 \mathrm{mV} / \mathrm{s}$ using cyclic voltammetry.
\end{abstract}

Keywords: hole transport layer (HTL); poly(ethylmethacrylate); reversible addition fragmentation chain-transfer (RAFT) polymerization; photovoltaic device; polycarbazole; precursor approach

\section{INTRODUCTION}

The interest in the development of organic photovoltaic devices (OPVs) based on the bulk heterojunction $(\mathrm{BHJ})$ concept of polymer solar cells (PSCs) has greatly increased because of their potential to reduce the price and negative environmental impact of Si-based solar cells. These PSCs have unique advantages of low cost, solution processable organic materials, and potential to be fabricated on large area and flexible substrates (Taranekar et al. 2007, Tria et al. 2011). One of the approaches that have been proposed to enhance the power cell efficiency (PCE) of PSCs is the development of effective hole (or electron) transport layer-materials (HTLM).
Unlike silicon or inorganic solar cells, OPVs usually require the photoactive layer to be sandwiched between two (2) different highly conductive layers, normally a metal such as Aluminum (Al) and a transparent oxide such as indium tin oxide (ITO) for effective charge extraction. The interface between the polymer active layer and the electrode performs a critical and essential role in determining the final performance of OPVs. Thus, inserting a functional interfacial layer can change the interface properties dramatically. A hole transport layer (HTL) between the polymer/anode interface such as poly $(3,4$ ethylene dioxythiophene):poly(styrene sulfonate) PEDOT:PSS film, for example, can be incorporated between the ITO and the photoactive layer. When 
a PEDOT:PSS film is inserted between the ITO anode and the photoactive layer, an improvement in the current at open circuit voltage $\left(\mathrm{V}_{\mathrm{oc}}\right)$ is observed (Tria et al. 2011). Although PEDOT:PSS has been commonly used to improve the performance of OLEDs and OPVs, the acidic and insulating nature of PSS imposes problems in terms of device stability. Also, the large particle size of PEDOT can induce the degradation of the layer because of the aggregation of particles causing defects. The high costs and low electrical conductivity deterred the use of PEDOT:PSS as HTL in addition to its acidity, which causes the degradation of the OSC device (Lim et al., 2012).

This study explores a new class of material that can potentially be used as an alternative HTLM in an organic photovoltaic device. This material is a polymer methacrylate containing cross-linked/ conjugated carbazole (for higher conductivity) electrodeposited on ITO. Specifically, we optimized the formation of the cross-linked polycarbazole film on ITO by preparing a prepolymer with uniform molecular weight distribution using RAFT polymerization, electrodepositing the polymer to form a uniform smooth film covering on the ITO surface, controlling its thickness, and demonstrating its electro-optical compatibility. Moreover, we examined the compatibility of the cross-linked polycarbazole film as potential HTLM material between ITO and an active layer of PCDTBT/ PCBMin a bulk-heterojunction configuration. Furthermore, we fabricated a PV cell with the film as HTLM between ITO and the active layer, and assessed the I-V characteristic of the PV cell under darkness and light illumination to determine its feasibility as HTL for solar cell applications.

\section{MATERIALS AND METHODS}

Preparation of Polymer Methacrylate Containing Cross-linked Polycarbazole. The synthesis of the carbazole monomer (CzEMA) was taken from the work of Taranekar et al. (2007) while the preparation of the prepolymer poly(CzEMA) by RAFT using 4-cyanopentanoic acid dithiobenzoate (CPDB) was according to the work of Pontillas et al., (2018). Electropolymerization on ITO by cyclic voltammetry (CV) was used in cross-linking the carbazole pendants in the polymer. Deposition on ITO-coated glass substrate was done from $-0.2 \mathrm{~V}$ to $+1.6 \mathrm{~V}$. The film produced was then washed with 1,2 -dichlorobenzene then dried at $60^{\circ} \mathrm{C}$ in vacuum.

Materials' Characterization. Several properties of the film were first satisfied such as: (1) uniform distribution of molecular weight $(\mathrm{Mw})$ and polydispersity index (PDI) of the prepolymer, (2) smoothness and uniform coverage of electrodeposited film on ITO substrate, (3) optimized film thickness, and (4) electrooptical compatibility, before application in a prototype device.

Uniform Molecular Weight and PDI.. The prepolymer $\underline{4}$ was synthesized using the RAFT polymerization technique. The $\mathrm{Mw}$ and PDI were determined by gel permeation chromatography (GPC).

Smoothness and Uniform Coverage. The effect of scan rate on smoothness of film was studied and thus, variable scan rates $(25 \mathrm{mV} / \mathrm{s}, 50 \mathrm{mV} / \mathrm{s}, 100$ $\mathrm{mV} / \mathrm{s}$ ) and different solvents (dichloromethane, dichlorobenzene, chloroform) were used during electropolymerization. Dichlorobenzene was used for washing the electrodeposited film to remove undissolved polymers. Morphological properties of the electrodeposited film were assessed using Atomic Force Microscopy (AFM).

Film Thickness and Number of Scan Cycles. The thickness of an HTL (suggested was 80-100 nm in order to transport holes effectively in OPVs) was measured by varying the number of scan cycles $(5,10$, $15,25)$ during electropolymerization. Approximate thicknesses of the electrodeposited polymer films on ITO-glass substrate were obtained by modelling and measurement analysis using the DeltaPsi software of the ellipsometer at a spectral range: $440-1000 \mathrm{~nm}$.

Electro-optical Properties. After assessment of the properties of the film for use as HTL, its electrooptical characteristics were evaluated. In order to evaluate the potential of the electropolymerized carbazole as HTL and as an alternative to PEDOT:PSS, highest occupied molecular orbital (HOMO) and lowest unoccupied molecular orbital (LUMO) approximations were done. HOMO was determined using the oxidation potential $\left(\mathrm{E}_{\mathrm{ox}}\right)$ obtained from the cyclic voltammogram and equation 1 (Crespilho et al., 2006) was used.

$$
\mathrm{HOMO}=\mathrm{E}_{\mathrm{ox}}+4.4
$$

Moreover, the optical bandgap $\left(\mathrm{E}_{\mathrm{g}}\right)$ of the electropolymerized carbazole was calculated by determining the onset of absorption $(\lambda)$ from the UV-Vis spectrum and substituting the value in equation 2.

$$
\mathrm{E}_{\mathrm{g}}=\mathrm{hc} / \lambda
$$

Thus, the LUMO was derived indirectly from the equation,

$$
\mathrm{LUMO}=\mathrm{HOMO}-\mathrm{E}_{\mathrm{g}} .
$$


UV-Vis absorption spectra were obtained from 900 $\mathrm{nm}-400 \mathrm{~nm}$ wavelength. Cyclic voltammograms were recorded at varying scan rates and were performed in a conventional three-electrode cell.

Device Fabrication. A prototype solar cell device was fabricated in this study in order to assess the photovoltaic properties of the synthesized polymer being used as a potential hole transport layer (HTL).

Preparation of the ITO Substrate. In preparing the patterned substrate, a Scotch brand clear tape was placed on the ITO surface to serve as a mask against acid etching. The etchant solution was prepared $\left(20 \% \mathrm{w} / \mathrm{v} \mathrm{HCl}, 5 \% \mathrm{w} / \mathrm{v} \mathrm{HNO}_{3}\right)$ and was heated at $70^{\circ} \mathrm{C}-75^{\circ} \mathrm{C}$. Then, the unmasked portion of the ITO was etched by dipping the substrate for 90 seconds in the etchant. After etching, the substrate was washed with 50\% deionized (DI) water and propanol. The tape was removed from the substrate and was sonicated in propanol for 10 mins. Cleaning the ITO-coated glass substrate was done by sonication for 15 minutes in a series of solvents; chloroform, acetone and ethanol, each for 5 minutes. Then, it was dumped rinse by washing with distilled water and then dried in oven at $110^{\circ} \mathrm{C}$.

Electrodeposition of the HTL 5. The HTL was deposited by first preparing the precursor polymer 4. A $50 \mathrm{mg}$ of $\underline{4}$ was dissolved in $50 \mathrm{~mL}$ of $0.1 \mathrm{M}$ TBAH electrolyte solution [dichloromethane (DCM), chlorobenzene (CB), or chloroform (CF) as the electrolyte solvent] and was sonicated until there was complete dissolution of the polymer. Three different scan rates $(5 \mathrm{mV} / \mathrm{s}, 25 \mathrm{mV} / \mathrm{s}, 50 \mathrm{mV} / \mathrm{s})$ from $-0.2 \mathrm{~V}$ to $1.6 \mathrm{~V}$ were used to electropolymerize the polymer on ITO-coated glass substrate. It was then washed with 1,2-dichlorobenzene to remove the undissolved polymers on the film and dried at $60^{\circ} \mathrm{C}$ in vacuum to remove excess solvents.

Deposition of the Active Layer. The active layer was deposited by first preparing the donor material by dissolving 8 mg of PCDTBT (Poly $N-9$ '-heptadecanyl2,7-carbazole-alt-5,5-(4',7'-di-2- thienyl-2', 1',3'benzothiadiazole) in $2 \mathrm{~mL}$ of chlorobenzene at $80^{\circ} \mathrm{C}$. Then, $32 \mathrm{mg}$ of PCBM (Phenyl-C61-butyric acid methyl ester), which was used as the acceptor material was blended with PCDTBT solution (1:4 ratio). It was stirred for 60 hours at $80^{\circ} \mathrm{C}$ to allow complete dissolution. Prior to spin coating, the solution was filtered through $0.45 \mu \mathrm{m}$ PTFE filter and then $20 \mu \mathrm{L}$ of the blended solution was spin coated at $3000 \mathrm{rpm}$ for 60 seconds until the entire substrate was covered with the active layer.

Thermal Deposition of the Aluminum Metal. Deposition of aluminum metal on the substrate with
80-120 nm thickness was carried out in vacuum in a thermal evaporator at $1 \times 10^{-5}$ mbar. After evaporation, it was allowed to cool in the evaporator before removal and was placed in a desiccator before testing.

Determination of Current at $V_{o c}$. I-V curves of the fabricated prototype solar cell device were plotted using EChem software of EDAQ. I-V curves were obtained (with and without illumination from a halogen lamp) in order to know if the device will have diode properties like that of a typical solar cell. Current at open circuit voltage $\left(\mathrm{V}_{\text {oc }}\right)$ were determined at different deposition rates $(25,50,100 \mathrm{mV} / \mathrm{s})$ and cycles $(5,10,15,25)$ and the optimized current at open circuit voltage was determined.

\section{RESULTS AND DISCUSSION}

Ethyl methacrylate with carbazole groups (Fig. 1) was successfully polymerized via (RAFT) to produce precursor polymers with controlled MW (16,017 \pm $995 \mathrm{~g} / \mathrm{mole}$ ) and narrow polydispersity index (PDI $=1.18 \pm 0.01$ ) as determined by GPC (compared with $\mathrm{PDI}=2.13 \pm 0.32$ through free radical polymerization). Cross-linking of the electroactive carbazole pendants in the polymer was done via $\mathrm{CV}$ on ITO-coated glass slide as working electrode in a three-electrode cell. The attachment of carbazole groups to the polymer backbone improved monomer availability and rendered them more soluble to organic solvents during electropolymerization. Thus, a smoother film ( $\mathrm{rms}=52 \mathrm{~nm}$ ) was obtained (see Fig.2A1-A2) compared to electropolymerization directly from carbazole monomers in solution ( $\mathrm{rms}$ $=121 \mathrm{~nm}$ ). By varying the electropolymerization parameters such as deposition rates $(25,50,100$ $\mathrm{mV} / \mathrm{s})$, number of cycles $(5,10,15,25)$ at $50 \mathrm{mV} / \mathrm{s}$, and use of different organic solvents (DCM, CB and CF), morphological features of the electrodeposited films were improved and optimized. Lower deposition rates $(25 \mathrm{mV} / \mathrm{s})$ allowed ordered nucleation on the electrode surface and more ordered structure of polymer chain growth during electropolymerization which led to the lower surface roughness $(\mathrm{rms}=$ $4.532 \mathrm{~nm}$ compared to $\mathrm{rms}=40.05 \mathrm{~nm}$ for 100 $\mathrm{mV} / \mathrm{s}$ ) and smoother film (See Fig. 2C1-C3). Among the solvents used, CF ( $\mathrm{rms}=6.14 \mathrm{~nm}$ ) proved to be the most effective, producing smoother surface with small domains and homogeneous topography with less pinholes compared to samples prepared in CB $(\mathrm{rms}=29.47 \mathrm{~nm})$ and DCM $(\mathrm{rms}=35.45 \mathrm{~nm})$. See Fig.2 B1-B3. A crack-free film was also observed with a roughness value lower than the calculated rms for CB and DCM. Also, films of various thicknesses were made by controlling the number of scan cycles. Spectroscopic ellipsometry was used to measure the thickness of the films and as the number of cycles 
increased, so did the thickness ( 5 cycles gave an rms of $53.38 \mathrm{~nm}$ while 25 cycles gave an rms of $150.48 \mathrm{~nm}$ ).

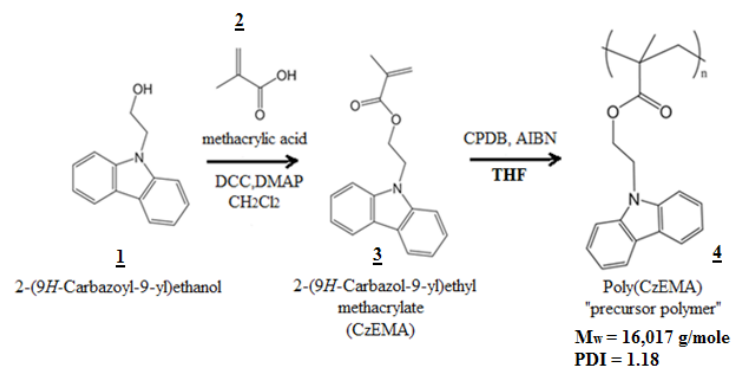

Figure 1. Synthesis of "Precursor Polymer" poly(CzEMA) by RAFT Polymerization.

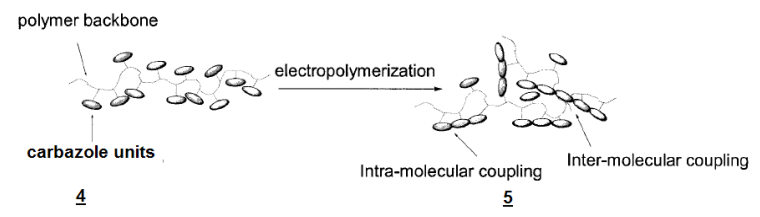

Scheme 1. Synthesis of $\mathbf{5}$ using the Precursor Approach (Fulghum et al., 2008).
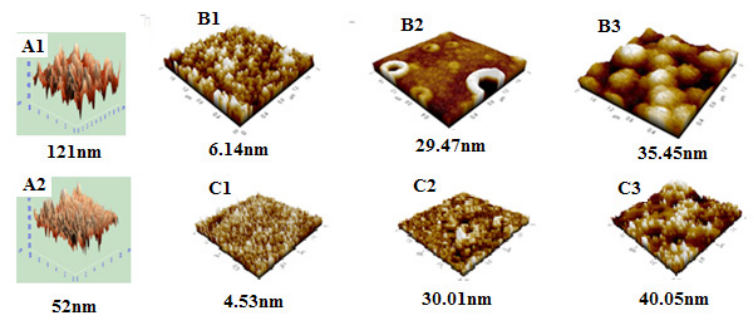

Figure 2. AFM Profile and Measured Roughness of the Film in rms (A1-A2) Straightforward vs Cross-linked, (B1-B3) Electropolymerization Solvents CF, CB, $D C M$; and (C1-C3) scan rates $(25,50,100 \mathrm{mV} / \mathrm{s})$.

From the evaluation of the electro-optical properties, the work function $\left(\Phi_{\mathrm{w}}\right)$ of e-polyCzEMA was $5.18 \mathrm{eV}$, which is centrally located between the work function of ITO and the HOMO energy level of PCDTBT. This $\Phi_{\mathrm{w}}$ was close to the $\Phi_{\mathrm{w}}$ of PEDOT:PSS (5.0$5.1 \mathrm{eV})$, the most commonly used hole transfer layer (HTL) in OSC devices. Because the values of $\Phi_{\mathrm{w}}$ of these HTLs were in the same range of 5.0-5.2, it can be considered that they have similar minimum energies required to remove an electron from their surfaces. Thus, this approximation suggested that the $\Phi_{\mathrm{w}}$ of the electropolymerized carbazole polymer was aligned with the HOMO (5.3-5.55 eV) of PCDTBT in the active layer. This energy band structure meant that the holes generated from the dissociation of photo-generated excitons can make a convenient transportation from PCDTBT to the ITO anode. Consequently, from all these electrooptical data, the e-polyCzEMA film would function as a good hole extracting and transporting layer in PCDTBT-based OSC devices.
The UV-Vis spectra indicated that the deposited film was almost non-absorbing or highly transparent in the visible region in the range of 450-600 nm. Transmittance values were approximately more than $90 \%$, comparable with transmittance of commonly used HTL, PEDOT:PSS, which is usually $88 \%$ at $450-800 \mathrm{~nm}$ (Bejbouji et al. 2010) Thus, e-polyCzEMA film can be considered suitable for use as HTL layer in OSC devices.

Prototype OSC device was fabricated using bare ITO only compared to ITO with electrodeposited polyCzEMA (e-polyCzEMA) as HTL (see diagram and prototype device in Fig. 3A-3B). Both devices presented non-linear I-V characteristics in both the forward and the reverse polarizations, showing properties of a diode. However, a larger shift along the negative $y$-axis of the device with ePCzEMA as HTL was observed upon light illumination, which suggested that the work function of e-polyCzEMA was well aligned with the HOMO of the active layer, forming an ohmic contact unlike the device having no HTL that led to mismatch of work functions. Consequently, I-V characteristics of other fabricated prototype solar cell devices was assessed with the variation of number of cycles $(5$, $10,15,25$ cycles) at deposition rate of $25 \mathrm{mV} / \mathrm{s}$ during electropolymerization. PolyCzEMA-based devices electro-deposited with 15 and 25 cycles with approximate thickness of 125 and $150 \mathrm{~nm}$ respectively, had better responses on their I-V curves; thus, indicating better performance. Variation in the deposition rate showed that e-polyCzEMA HTL films deposited at lower rate $(25 \mathrm{mv} / \mathrm{s})$ are more homogeneous and more compact. As a consequence, the lower roughness of such transporting layer surface led to higher optoelectronic performance as depicted by its I-V curve. Comparison of measurements of the current at open circuit voltage during illumination (see Fig. 3C-3D) showed that the cell with HTL produced 70 times larger current at $\mathrm{V}_{\mathrm{oc}}$ than that of the cell without HTL.

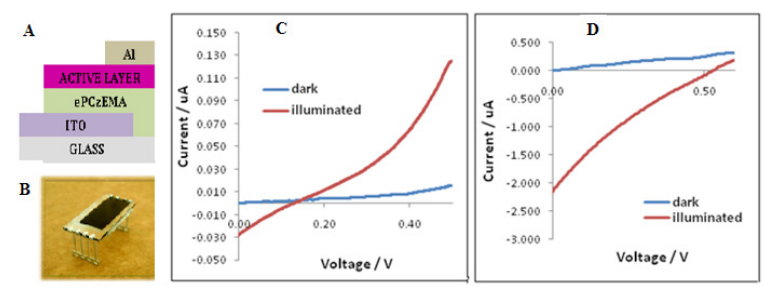

Figure 3. (A-B) Diagram and Picture of Prototype Cell and its Response to Light Illumination Represented by the I-V graphs (C) no HTL and (D) with HTL. HTL is ePCZEMA. 


\section{CONCLUSION}

RAFT synthesized PCzEMA, which is consequently crosslinked on deposition to ITO by $\mathrm{CV}$, with optimization of its film properties such as smoothness and thickness by using appropriate deposition rates, number of scan cycles and electropolymerization solvents, can be used as potential HTL in bulk heterojunction photovoltaic devices. This works well with PCDTBT/PCBM as active layer leading to increase in the current at open circuit voltage on illumination by 70 times (and at PCE of 293 times) compared to a cell without HTL. It can therefore substitute for PEDOT-PSS as HTLM overcoming some of its disadvantages such as acidity, low conductivity and aggregation tendency leading to film defects.

\section{ACKNOWLEDGEMENTS}

We would like to acknowledge the funding support of the Philippine Council for Industry, Energy and Emerging Technology Research and Development (PCIEERD) of the Department of Science and Technology (DOST).

\section{REFERENCES}

Bejbouji H, Vignau LL, Miane JL, Dang MT, Oualim EM, Harmouchi M, Mouhsen A. Polyaniline as a hole injection layer on organic photovoltaic cells. Sol Energy Mater Sol Cells. 2010;94(2):176-181. doi:10.1016/j.solmat.2009.08.018.

Crespilho FN, Zucolotto V, Siqueira JR, Carvalho AJF, Nart FC, Oliveira ON. Using electrochemical data to obtain energy diagrams forn layer-bylayer films from metallic phthalocyanines. Int J Electrochem Sci. 2006;1:151-159.

Fulghum TM, Taranekar P, Advincula RC. Grafting Hole-Transport Precursor Polymer Brushes on ITO Electrodes: Surface-Initiated Polymerization and Conjugated Polymer Network Formation of PVK. Macromolecules 2008;41(15):5681-5687.

Lim TH, Oh KW, Kim SH. Effect of self-assembly supramolecules on the electrical properties of polyaniline based hole transport layer. Synth Met. 2012;162(3-4):268-275. doi:10.1016/j. synthmet.2011.12.002.

Pontillas SM, Sumera F, Advincula R. Reversible addition fragmentation chain transfer (RAFT) polymerization of poly(ethylmethacrylate) with pendant carbazole groups. KIMIKA. 2018; 29:4150.
Taranekar P, Fulghum T, Patton D, PonnapatiR, Clyde G, Advincula RC. Investigating carbazole jacketed precursor dendrimers: sonochemical synthesis, characterization, and electrochemical crosslinking properties. J Am Chem Soc. 2007;129(41):1253712548. doi:10.1021/ja074007t.

Tria MC, Liao K, Nigel A, Curran S, Advincula RC. Electrochemically crosslinked surface-grafted PVK polymer brushes as a hole transport layer for organic photovoltaics. J Mater Chem 21. 2011;21:1026110264. 